\title{
Editorial
}

\section{Decentralized Modeling, Analysis, Control, and Application of Distributed Dynamic Systems}

\author{
Ning Cai, ${ }^{1,2}$ Roberto Sabatini, ${ }^{3}$ Xi-Wang Dong, ${ }^{4}$ M. Junaid Khan, ${ }^{5}$ and Yao $\mathrm{Yu}^{6}$ \\ ${ }^{1}$ School of Electrical Engineering, Northwest University for Nationalities, Lanzhou, China \\ ${ }^{2}$ Key Laboratory of National Language Intelligent Processing, Gansu, China \\ ${ }^{3}$ School of Engineering-Aerospace and Aviation Discipline, RMIT University, Melbourne, VIC, Australia \\ ${ }^{4}$ School of Automation Science and Electrical Engineering, Beihang University, Beijing, China \\ ${ }^{5}$ School of PN Engineering, National University of Sciences and Technology, Islamabad, Pakistan \\ ${ }^{6}$ School of Automation, University of Science and Technology Beijing, Beijing, China \\ Correspondence should be addressed to Ning Cai; caining91@tsinghua.org.cn
}

Received 29 November 2016; Accepted 29 November 2016

Copyright (C) 2016 Ning Cai et al. This is an open access article distributed under the Creative Commons Attribution License, which permits unrestricted use, distribution, and reproduction in any medium, provided the original work is properly cited.

Distributed dynamic systems are large-scale systems constituted by numerous subsystems deployed in distributed manner. In many cases, there are three fundamental characteristics for these subsystems. The first characteristic is autonomy. The subsystems are usually autonomous or semiautonomous and can often exist and operate independently. The second characteristic is homogeneity. The subsystems often share similarity and play relatively equal roles. The last and most important characteristic is interactivity. The subsystems should connect to a network topology collectively, such that they are able to communicate by exchanging information or substance and cooperate with each other.

Since the beginning of the current century, studies on the modeling, simulation, analysis, and synthesis about distributed dynamic systems have gradually formed a highlighted direction in the area of control and systems science, meanwhile receiving more and more attention from increasing number of scientific and application disciplines. Relevant research includes time and frequency domain methods, state space analysis, filtering, prediction, optimization, system identification, robust control, and stochastic control, just to mention a few. This new trend can be attributed to the joint advancements of scientific methodology, engineering technology, and control theory. Along with the development of modern computing technique, there appear signs for the scientific methodology in systems science to return back to reductionism from holism, especially for modeling and analysis. In the meantime, mankind is rapidly stepping into a networked world. The notion of complex and dynamical networks keeps on penetrating nearly all kinds of domains, ranging from communication networks, power networks, manufacturing networks, biological networks, and even social networks. For the networked distributed systems, the set of essential theoretical concepts of systems analysis and synthesis which are already fully developed on isolated systems such as stability, equilibrium, controllability/observability, and tracking control acquire new connotations and contents, which need to be extended profoundly. Moreover, methodologies based on networked configurations could also facilitate offering solutions to various difficult tasks. Nonetheless, extra complexity usually accompanies these systems due to the nonlinearities, distributed parameters and states, and high order. It is responsible and challenging for the control community to promote the advancement of both theories and technologies for modeling, analyzing, and controlling of networked distributed systems.

This special issue is composed of seven research articles, representing some of the recent advances around the theme of decentralized modeling, analysis, or synthesis on either directly controlling various distributed dynamic systems or utilizing these systems to solve other problems. Most of these articles seek solutions for engineering tasks with different 
backgrounds. For instance, among them, two papers deal with control strategies for maneuvering vehicles, two address the process control of steel plate manufacturing, one builds neuron circuits, and one paper concerns the control of brushless DC motors. A brief description of the papers follows.

In the paper "Decentralized Robust Adaptive Output Feedback Stabilization for Interconnected Nonlinear Systems with Uncertainties," Q. Yang et al. synthesized a strategy of decentralized robust adaptive output feedback stabilization for a class of large-scale nonlinear systems comprised of interconnected subsystems with uncertainties, based on the adaptive nonlinear damping technique. Their scheme is implemented by integrating a robust output feedback controller with a state observer, both being decentralized and adaptive, and can practically realize stabilization or tracking.

L. Yuan et al. investigated the synchronization reliability of neuronal circuits connected with ring type in the paper "Synchronization of Neuronal Circuits with Ring Connection on PSpice." In this paper, analog signals are converted into digital signals, so that the synchronization rhythm and phase synchronization can be detected from the sampled time series for membrane potential of Hindmarsh-Rose neurons. It is confirmed by the authors that the synchronization behavior and stability of the network depend on the coupling intensity greatly, and it never means stronger intensity can ensure stable synchronization under bursting state.

S. Shen and P. Zhou proposed a scheme for chaos synchronization of fractional-order brushless DC motors in the paper entitled "Synchronization of the Fractional-Order Brushless DC Motors Chaotic System.” The previous work of the authors has shown that, under certain conditions, the nonlinear model of brushless DC motors is a chaotic system of fractional-order, and synchronization is required between different systems. In the paper, the authors designed a feedback controller based on relative output information to ensure asymptotic synchronizations and theoretically validated its effectiveness.

In the paper "A Novel Relative Navigation Control Strategy Based on Relation Space Method for Autonomous Underground Articulated Vehicles," F. Dou et al. designed a scheme for relative navigation control of trackless articulated underground vehicles based on the relation space method. A noting highlight of the technique is the employing of a self-organizing, competitive neural network to identify the information of the surrounding space of the vehicles, which is important for programming an optimal driving direction with the known spatial geometric relationships.

V. Pshikhopov et al. presented a decentralized control scheme of homogeneous vehicles in obstructed environment. The information of obstacles is transformed into repellers based on artificial potential field approach, with the unstable modes being exploited to dynamically produce the appropriate repelling forces at different positions.

F. Zhang et al. developed a distributedly deployed technological package of equipment for the intermediate cooling control during hot rolling of steel plate in production line in the paper "Development of Intermediate Cooling Technology and Its Control for Two-stand Plate Rolling." The overall framework of the package comprehensively combines feedforward, feedback, and adaptive algorithms.

The paper "Rolling Force Prediction in Heavy Plate Rolling Based on Uniform Differential Neural Network" by F. Zhang et al. provided a uniform differential evolution algorithm with fine performance in high dimensional function optimization for optimizing the structure and weight value of neural network. The algorithm was used to improve the accuracy of rolling force prediction in the process control system of medium and heavy plate.

\section{Acknowledgments}

We would like to thank all the authors and co-authors who submitted their work for consideration to this special issue as well as the anonymous reviewers for their efforts to ensure the standard of published papers.

Ning Cai

Roberto Sabatini Xi-Wang Dong M. Junaid Khan Yao Yu 


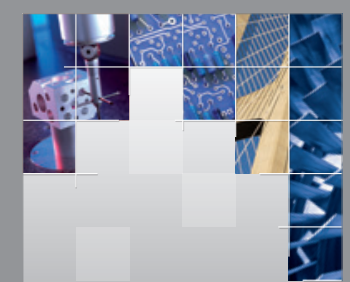

\section{Enfincering}
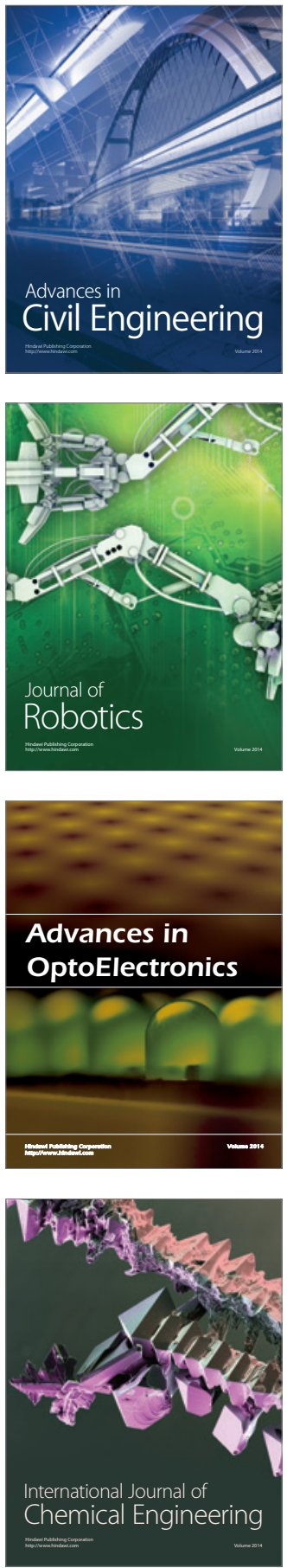

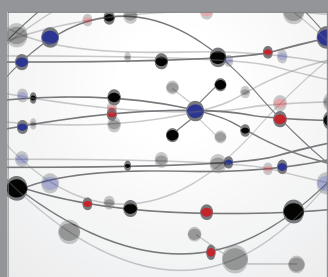

The Scientific World Journal

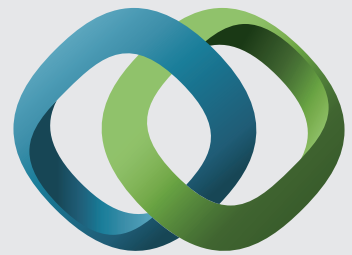

\section{Hindawi}

Submit your manuscripts at

http://www.hindawi.com
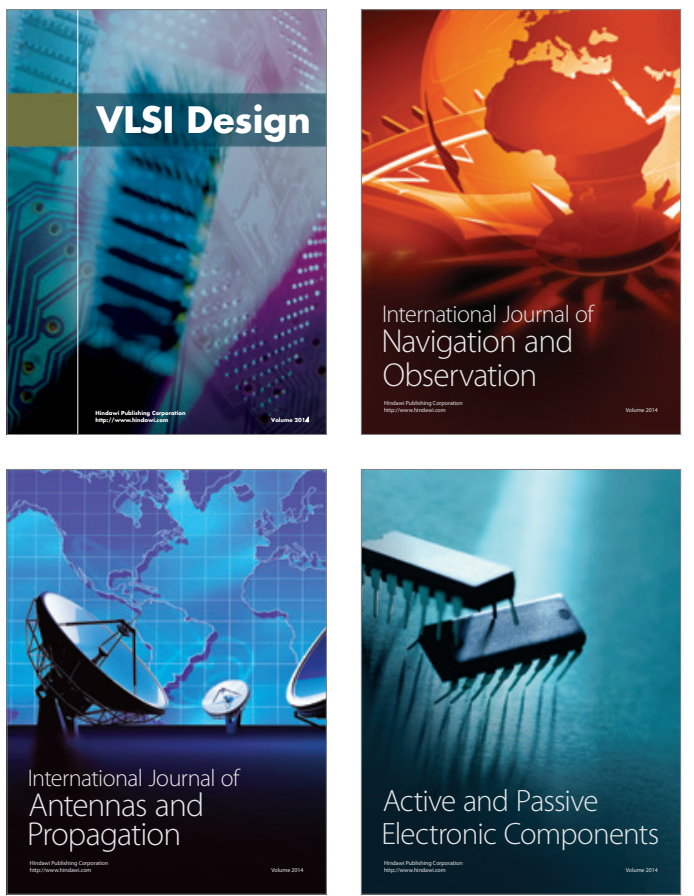
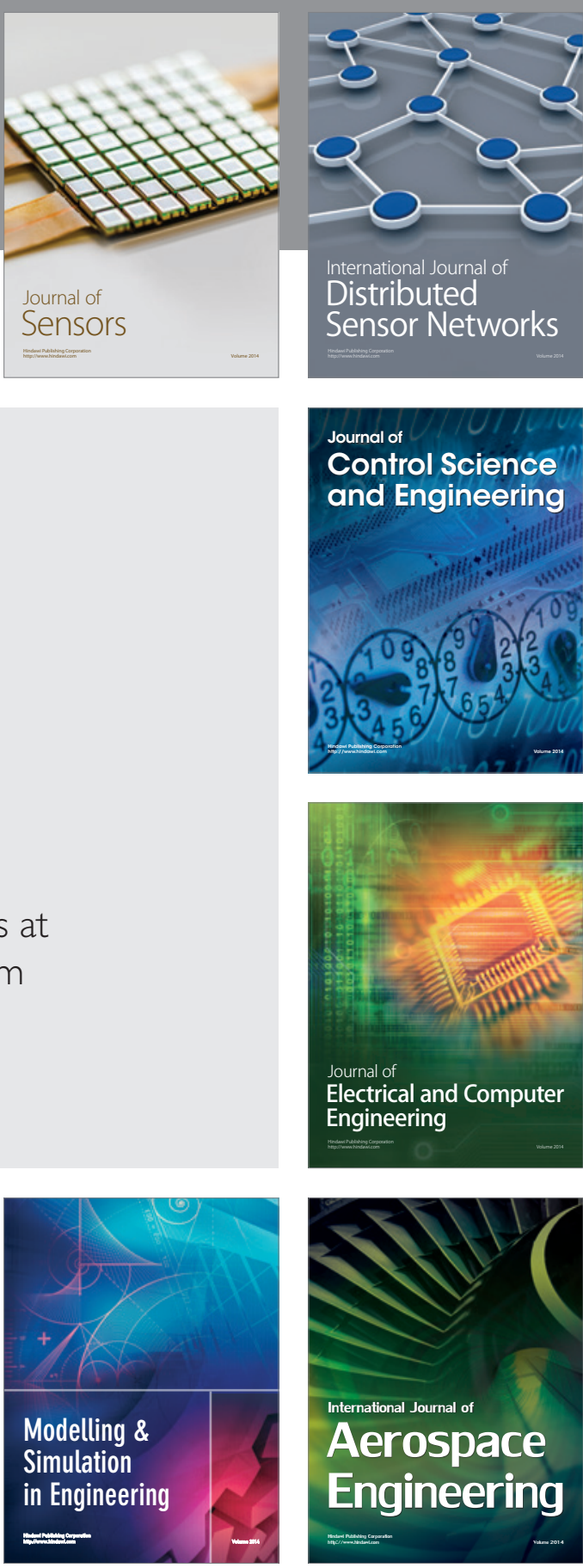

International Journal of

Distributed

Sensor Networks

Journal of

Control Science

and Engineering
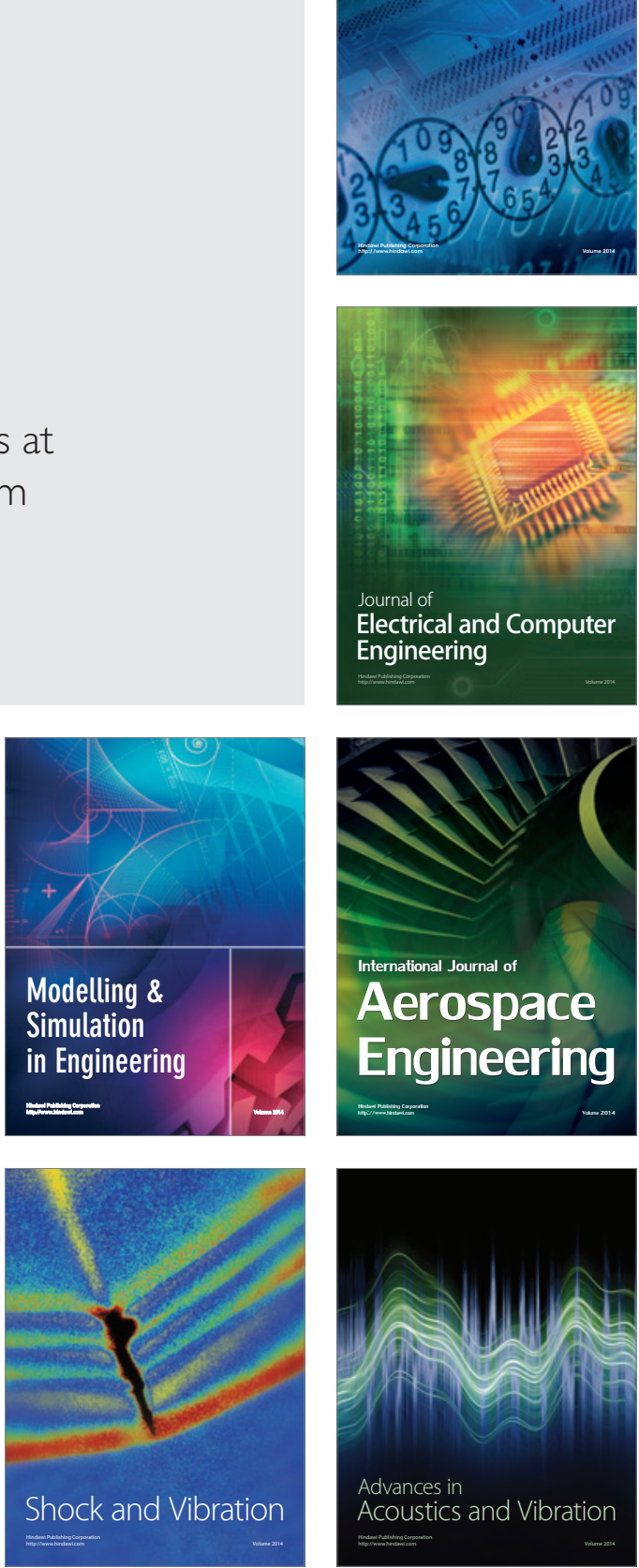